\title{
Anthelmintic Flubendazole and Its Potential Use in Anticancer Therapy
}

\author{
Kristýna Čáňová ${ }^{1}$, Lucie Rozkydalová2 ${ }^{2}$ Emil Rudolf 1 ,*
}

\begin{abstract}
Flubendazole is a widely used anthelmintic drug belonging to benzimidazole group. The molecular mechanism of action of flubendazole is based on its specific binding to tubulin, which results in disruption of microtubule structure and function, and in the interference with the microtubule-mediated transport of secretory vesicles in absorptive tissues of helminths. The microtubule-disrupting properties of benzimidazole derivatives raised recently interest in these compounds as possible anti-cancer agents. In this minireview flubendazole effects towards selected human malignant cells including myeloma, leukemia, neuroblastoma, breast cancer, colorectal cancer and melanoma are discussed along with basic data on its pharmacokinetics, metabolism and toxicity.
\end{abstract}

\section{KEYWORDS}

flubendazole; benzimidazole carbamate; anti-cancer treatment; melanoma; microtubules; mitotic catastrophe

\section{AUTHOR AFFILIATIONS}

${ }^{1}$ Department of Medical Biology and Genetics, Charles University, Faculty of Medicine in Hradec Králové, Czech Republic

2 Department of Pharmacology, Charles University, Faculty of Pharmacy in Hradec Králové, Czech Republic

* Corresponding author: Charles University, Faculty of Medicine in Hradec Králové, Šimkova 870, 50038 Hradec Králové, Czech Republic; e-mail: rudolf@lfhk.cuni.cz

Received: 18 January 2017

Accepted: 16 March 2017

Published online: 12 April 2017

Acta Medica (Hradec Králové) 2017; 60(1): 5-11

https://doi.org/10.14712/18059694.2017.44

(c) 2017 The Authors. This is an open-access article distributed under the terms of the Creative Commons Attribution License (http://creativecommons.org/licenses/by/4.0), which permits unrestricted use, distribution, and reproduction in any medium, provided the original author and source are credited. 


\section{INTRODUCTION}

Cancer continues to be one of the leading causes of morbidity and mortality worldwide. Although there are numerous researches about anticancer therapy, with many lead candidates at various stages of preclinical or clinical research, only $5 \%$ of compounds originally entering Phase I clinical trials are in the end approved (1).

Historically, discovery of novel antineoplastic agents was essentially linked with pharmacological and biochemical analyses of diverse natural sources including microorganisms, animals and plants, often followed by total chemical synthesis or modification of identified individual compounds. This approach has been lately complemented by screening assays of libraries of all known and identified compounds with aim of selection of potentially suitable candidates for further testing. Although these mentioned strategies are still used, they suffer from several potential drawbacks including time cost, financial burden and laboriousness. New strategies are therefore needed to optimize the development of new, potent antineoplastic compounds. One of the promising strategies is drug repositioning (or repurposing). Drug repositioning is the process of searching for new indications of existing drugs (2). This is potentially efficient approach since many existing drugs are of established formulations produced by standardized manufacturing methods and their pharmacokinetic, pharmacodynamic and toxicity aspects are well known, thus reducing the chance of their future failure due to adverse side effects. Moreover, reduced cost of development as well as the shortened overall time required for approval can also be considered an advantage $(1,3,4)$. These all factors contributed to the fact that to the date, there are at least 46 approved drugs already repositioned for new therapeutic uses as indicated in literature (5).

One of the potential pharmacological candidate for repurposing is flubendazole. Flubendazole ([5-(4-fluorobenzoyl)-1H-benzimidazole-2-y1]-carbamic acid methyl ester) belongs to the class of synthetic anthelmintic compounds (Fig. 1) which was found by Janssen company in 1970s.

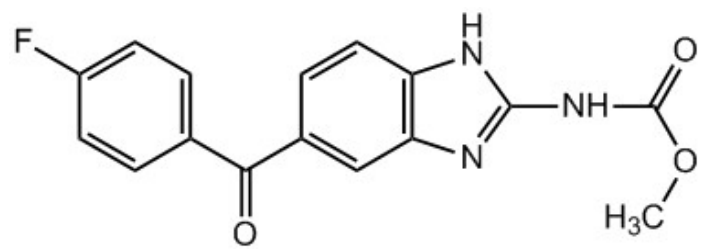

FIG. 1: Structure of flubendazole.

This compound belongs to the group of benzimidazole carbamates - drugs widely used in human and veterinary medicine against parasitic worms. The members of this group including flubendazole show wide range of efficiency towards gastrointestinal nematode infections in swine, poultry and domestic animals, as well as against lungworms in swine. In these indications, flubendazole is usually administered over 3 days at doses of approximately $5 \mathrm{mg} / \mathrm{kg}$, but it is probably also efficient when given as a single dose at this same rate. In Europe, flubendazole is registered also for human use under the commercial name Fluvermal ${ }^{\ominus}$ for treatment of intestinal nematodes (4).

\section{MECHANISM OF ACTION}

The mechanism of action of benzimidazole carbamates encompasses their ability to specifically bind and interact with the microtubule subunit protein of $ß$-tubulin (6).

Microtubules, filamentous intracellular structures, are among main components of the eukaryotic cytoskeleton. They are composed of a single type of globular protein, called tubulin. Tubulin is a heterodimer consisting of two closely related polypeptides, $\alpha$-tubulin and $\beta$-tubulin. Accordingly, it has been found that benzimidazole carbamates generally interact with mammalian tubulin even if the affinity for a mammalian tubulin is weaker compared with the one of helminths (7). Since microtubule structures are very important for many vital functions of the parasite such as proliferation, mitosis, intracellular transport of organelles, maintenance of cell shape or cell locomotion, alteration of microtubular assembly and dynamics leads to the final destruction of the parasite $(6,8,9)$. In addition, benzimidazole carbamates may also inhibit energy metabolism of parasite cells. They cause disruption of transport and metabolism of glucose, resulting in energy and glycogen store depletion and loss of cellular motility. Even this process ultimately contributes to the death of the parasite $(10,11)$.

\section{PHARMACOKINETICS}

Benzimidazole carbamates including flubendazole are usually administered orally and absorbed through the gastrointestinal tract. Only small quantities of flubendazole are resorbed after oral treatment of pigs, rats, sheep, dogs and humans (12). Flubendazole is very poorly soluble in aqueous systems, which are found in the gastrointestinal tract, causing its low absorption to the bloodstream and thus very low bioavailability. Maximal plasma concentration levels of flubendazole in humans were lower than $5 \mathrm{ng} / \mathrm{ml}$ even after an oral dose of $2 \mathrm{~g}$. Its absorption is markedly increased if the substance is used immediately after a meal (13). More than $80 \%$ of an oral dose is excreted in feces and only very small amounts of unchanged drug (less than 0.1\%) are found in the urine. The half-life of flubendazole in tissues is $1-2$ days $(14,15)$.

Low bioavailability is the main limitation for use of flubendazole for treatment of many tumors thus its potential future use in oncology must be associated with modifications enhancing its availability. It was established that the dosage of the benzimidazole drug doesn't have an influence on the area under the concentration curves (AUC). For example dogs treated with fenbendazole with a single dose with a different range of 25 to $100 \mathrm{mg} / \mathrm{kg}$ had similar AUC level (16). Another study has found that the maximum concentration $\left(C_{\max }\right)$ of flubendazole observed after its administration is significantly higher when solution is used instead of suspension. Moreover it was demonstrated that hydroxypropyl- $\beta$-cyclodextrin significantly increases aqueous solubility of flubendazole, which resulted in the higher plasma $C_{\max }$ and enhanced its absorption and bioavailability in treated mice (17). Hydroxypropyl- $\beta$-cyclodextrin is commonly-used reagent for enhancing bioavail- 
ability of lipophilic drugs and can be used in both liquid and solid dosage forms (4).

\section{METABOLISM OF FLUBENDAZOLE}

There are two possible ways of flubendazole biotransformation. Absorbed flubendazole is during first-pass metabolism transformed in the liver, where hydrolysis of carbamate or reduction of ketone could take place. Ketoreduction to methyl [5-[(fluorophenyl)hydroxymethyl]-1H-benzimidazol-2-yl] carbamate was the major metabolic pathway verified in chickens and turkeys. Second metabolic pathway is carbamate hydrolysis to (2-amino-1H-benzimidazole-5-yl)(4-fluorophenyl)methanone, which is the major metabolic pathway in pigs. Products of both mentioned pathways are later converted to 2 -amino- $\alpha$-(4-fluorophenyl)-1H-benzimidazole-5-methanol. Metabolic pathways of flubendazole are shown in scheme (Fig. 2, 18).

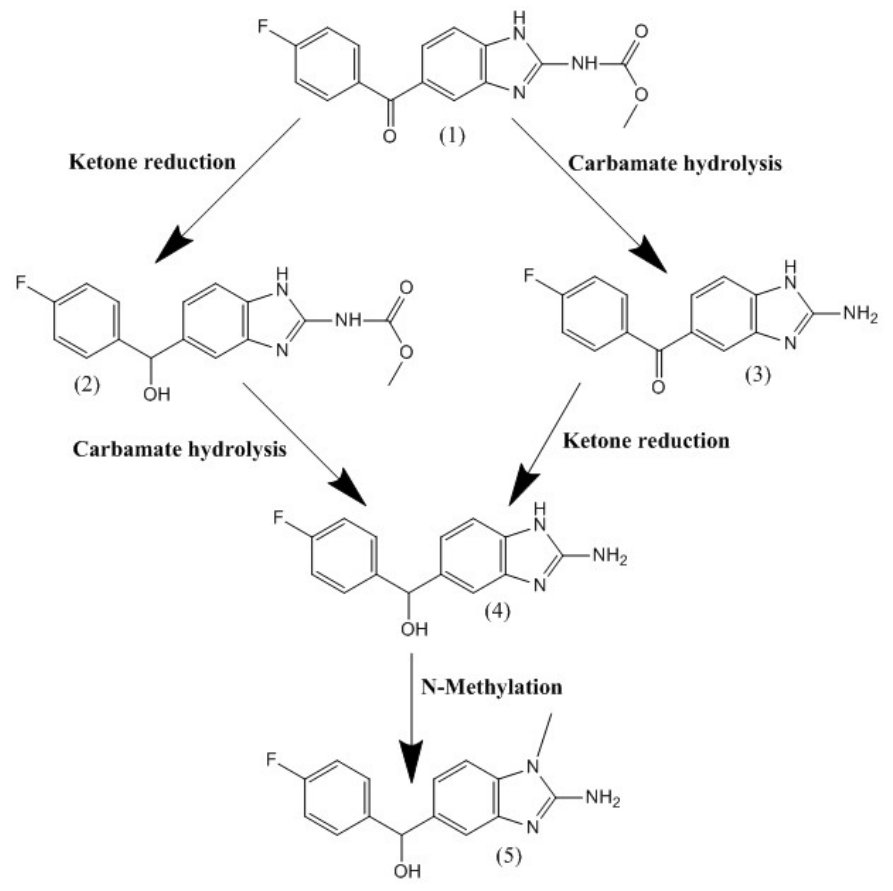

FIG. 2: Metabolic pathways of flubendazol (established by Van Leemput L. et al., 1991).

(1) - flubendazole

(2) - methyl-\{5-[a-hydroxy-a-(4-fluorophenyl) methyl]-1Hbenzimidazol-2-yl\} carbamate

(3) - (2-amino-1H-benzimidazol-5-yl)-4-fluorophenyl-methanone

(4) - 2-amino-a-(4-fluorophenyl)-1H-benzimidazole-5-methanol

(5) - 2-amino-a-(4-fluorophenyl)-1-methyl-1H-benzimidazole-5methanol

\section{TOXICITY}

The median lethal dose of flubendazole was explored in mice, rats and guinea pigs and was determined to have the value exceeding $2.56 \mathrm{~g} / \mathrm{kg}$ (12). In selected animal species flubendazole-dependent teratogenic effects as well as its influence on fertility were tested. In all these species, flubendazole was well tolerated. In pigs, flubendazole showed no teratogenicity and didn't have any effect on their fertility. Similarly, it did not affect reproductive ability or egg quality in hens and did not significantly diminish fertility in pheasants. Moreover, lack of any negative flubendazole-specific effect on reproductive performance was noted in the study with dogs. Finally, in rats fluebendazole failed to produce any embryotoxic, foetotoxic, or teratogenic effects.

In several in vitro assays on gene mutation in bacteria and yeast cells as well as in in vitro assays on DNA damage in Drosophila melanogaster flubendazole tested negative. A carcinogenicity study in Wistar rats reported no evidence of transformation potential of this compound (14). Furthermore, toxicity studies in humans showed that treatment with flubendazole of neurocysticercosis with doses 40 to $50 \mathrm{mg} / \mathrm{kg} / \mathrm{d}$ for 10 days and treatment of alveolar echinococcosis with doses $50 \mathrm{mg} / \mathrm{kg} / \mathrm{d}$ for 24 months produced no toxicity and adverse effects $(19,20)$.

\section{ANTICANCER ACTIVITY OF BENZIMIDAZOLE CARBAMATES IN PRECLINICAL MODELS}

A number of reports demonstrated ability of benzimidazole carbamates to inhibit polymerization of mammalian tubulin in vitro. These results raised the question of whether this class of compounds could inhibit tubulin polymerization in human cells too and if so whether such a targeted effect could lead in a wider context to their antiproliferative and/ or antitumor effects. First study to address this issue was published in 1985. It investigated a number of 5(6)-substituted methyl benzimidazole carbamate analogues and their activity against mouse leukemia cells L1210. In 1989, Robin et al. subsequently proved that benzimidazole carbamate albendazole is cytotoxic in hepatocellular carcinoma cell line Hep2G. Despite this early promising evidence, more thorough inquiry into antiproliferative potential of benzimidazole carbamates was realized almost after a decade only. In a panel of human, rat and mouse tumor liver cell lines albedazole at concentrations of $100 \mathrm{nM}$ and higher was found to inhibit proliferation of all tested cells which correlated with concentration-dependent changes in cell cycle distribution. Upon lower employed albendazole concentrations (up to $500 \mathrm{nM}$ ), exposed cells accumulated in G1 phase while higher albendazole concentrations (around $1000 \mathrm{nM}$ ) mediated in the same cells transition delay through $\mathrm{G} 2 / \mathrm{M}$ or mitosis. These in vitro observations were then by the same authors recapitulated in vivo using a xenographt model in which the growth of subcutaneous tumors induced by implantation of human tumor SKHEP-1 cells into nude mice was inhibited by the application of albendazole although at higher dose of $300 \mathrm{mg} / \mathrm{kg} /$ day only. Such a significant difference in effective albendazole concentrations in both models was attributed to the very high rate of metabolism of albendazole in mice and the poor blood supply to the studied tumor. Despite these observations, however, authors concluded that albendazole has a promising antitumor potential to be further investigated for the potential human use (21).

The anti-tumor efficiency of albendazole was also studied in a model of colorectal cancer. Albendazole and its 
main metabolite albendazole sulfoxide were found to potently inhibit growth and proliferation of human colorectal cell line HT-29, with the IC50 values of $0.12 \mu \mathrm{M}$ for albendazole and $2.35 \mu \mathrm{M}$ for albedazole-sulfoxide, respectively. Cytotoxicity of the same compound was also investigated in other colorectal cancer cell lines representing various stages of this malignancy (SW480, SW620, NCM460, Caco2 and HCT-8) where the most sensitive proved to be HCT-8 cells (IC50 $0.4 \mu \mathrm{M}$ ). Generally speaking, the main mechanism behind the observed effects of the employed compounds was induced arrest of cells at G2/M phase of the cell cycle and presence of caspase-3-mediated apoptosis (22). Furthermore, in nude mice HT-29 xenografts, regionally administered albendazole ( $150 \mathrm{mg} / \mathrm{kg} / \mathrm{i} . \mathrm{p}$.) proved its efficacy in peritoneal carcinomatosis (23).

In paclitaxel-resistant leukemic cells CEM/dEpoB300 treatment with albendazole induced significant cytotoxicity (IC values being as low as $0.32 \mu \mathrm{M}$ in CEM and $0.16 \mu \mathrm{M}$ in CEM/dEpoB300). Albendazole in this case stimulated massive depolymerization of microtubular network and activation of morphologically distinct apoptosis occurring via downregulated expression of BCL-2 and MCL-1 proteins, mitochondrial release of cytochrome $c$ and increased abundance of proapoptotic BAX as well as caspase-3 (24). Depolymerization of microtubules as a result of albendazole presence in the cell and resulting cell death was also confirmed in ovarian cancer cells sensitive (1A9) and resistant to paclitaxel (1A9PTX22) (25).

Another benzimidazole carbamate mebendazole was found effective in a number of in vitro models including adrenocortical carcinoma cell lines (26), non-small lung cancer cells (27), cholangiocarcinoma cancer cells (in vivo activity was noted too) (28), malignant melanoma cells (29), colon cancer cells $(30,31)$ and gastric cancer cells, where it could not only inhibit their growth but also migration and invasion (32).

\section{ANTICANCER ACTIVITY OF FLUBENDAZOLE IN PRECLINICAL MODELS}

Anti-tumor activity of flubendazole was first reported in leukaemia and myeloma cells originating from both established stabilized cell lines as well as from patients samples. At low and pharmacologically feasible $\mathrm{nM}$ concentrations, flubendazole induced mitotic catastrophe and cell death in malignant cells and delayed tumour growth in vivo. Mechanistically, flubendazole altered microtubule structure and inhibited tubulin polymerization by interacting with a site on tubulin similar to colchicine but distinct from that of Vinca alkaloids. A similar interaction with tubulin was also noted for mebendazole. However, other benzimidazole carbamate benomyl has been found to inhibit tubulin polymerization by interacting at a site distinct from both the colchicine and the Vinca domains. Thus, the actual mechanism of benzimidazoles-mediated inhibition of tubulin formation in exposed cells apparently varies within family members and may provide rationale for their putative usefulness in cases of recognized particular microtubule alterations in malignant cells.
Even more importantly, in cells resistant to vinblastine because of overexpression of P-glycoprotein full sensitivity to flubendazole was retained. In addition, flubendazole when supplied with both vinblastine and vincristine in vitro could reduce the viability of OCI-AML2 cells and delay tumor growth in a leukemia xenograft model more than either drug alone (33).

In a screen of a panel of 321 cell lines including cell lines from 26 cancer entities was neuroblastoma identified as another highly flubendazole-sensitive malignancy type. In the more refined screen aimed now on neuroblastoma model only, flubendazole displayed broad activity towards primary neuroblastoma cells obtained from five patients and a panel of 140 neuroblastoma cell lines with acquired drug resistance against major microtubule-binding compounds, with confirmed independence on major $A B C$ transporters $A B C B 1$ and $A B C G 2$ expressions. The anti-neuroblastoma activity of flubendazole involved p53-signalling where the MDM2 inhibitor and p53 activator nutlin-3 strongly potentiated the observed flubendazole effects. Further inquiry into mechanisms of flubendazole-induced apoptosis suggested PUMA to be a key mediator of the induced effects. In addition, a water-soluble flubendazole-(2-hydroxypropyl)- $\beta$-cyclodextrin preparation inhibited vessel formation and tumor growth in the chick chorioallantoic membrane model in vivo, thus attesting to the potential treatment potential of flubendazole in neuroblastoma and feasibility of its further testing (34).

In another recently published study, flubendazole inhibited proliferation of several breast cancer cell lines (MDA-MB-231, BT-549, SK-BR-3 and MCF-7) in dose- and time-dependent manner and was shown to delay tumor growth in xenograft models by intraperitoneal injection. Flubendazole specifically reduced CD44high/CD24low subpopulation of treated cells and suppressed the formation of mammosphere and the expression of several, stem cell phenotype-related genes comprising $c-m y c$, oct-4, sox-2, nanog and cyclin D1. Moreover, authors noted that flubendazole induced cell differentiation and inhibited cell migration. Among flubendazole-specific effects in breast cancer cells were also reduced expression of mesenchymal markers (i.e. $\beta$-CATENIN, N-CADHERIN and VIMENTIN) and an induced epithelial and differentiation marker (KERATIN 18). Observed antiproliferative effects of flubendazole were further associated with arrested cell cycle at G2/M phase and induced monopolar spindle formation through inhibited tubulin polymerization. In combined regimens, flubendazole enhanced cytotoxicity of standard therapeutic drugs fluorouracil and doxorubicin against breast cancer cells (35).

Besides exerting antiproliferative effects in breast cancer cells via inhibition of tubulin polymerization and cell cycle arrest, flubendazole proved to be a potent inducer of reactive oxygen species (ROSs) and could activate autophagy as demonstrated by Zhanq et al. Their in-silico analysis and experimental validation provide evidence on flubendazole as a compound capable of targeting the autophagy-related protein 4B(ATG4B) in MDA-MB-231 cell line with a promising potential for further testing of its usefulness in case of triple negative breast cancer therapy (36). 
Flubendazole-based antiproliferative activity was also investigated on the small panel of colorectal cancer cell lines. Predictably, flubendazole inhibited the growth of these cells by the cell cycle arrest in the G2/M phase and also potentiated the effect of paclitaxel in this cellular model (22). In colorectal cancer SW480 and SW620 cells, low concentrations of flubendazole were cytotoxic as demonstrated by the presence of disrupted microtubular network, aberrant mitotic spindles and cell cycle perturbations accompanied by changes in CYCLIN D and B1 levels. Resulting activation of CASPASE-2 and CASPASE 3/7 as well as PARP cleavage lead to mitotic catastrophe and appearance of premature senescence phenotypes with typical giant multinucleated cells positive for SA- $\beta$-galactosidase staining. Although significant in their extent, these flubendazole-related effects were only temporary in their appearance and exposed cells were able to recover from them. This observation raised several important questions (such as the issue of longer term response rates of cancer cells to this compound as well as the nature of mechanisms enabling the rescue of cells undergoing mitotic catastrophe and/or premature senescence etc.) prompting the need of further studies in this field (37).

In our laboratory, we are investigating effects of flubendazole on malignant melanoma. Melanoma is the most aggressive form of skin cancer, with a high propensity to metastasize (38). Most metastatic melanoma patients fail to respond to available therapy, underscoring the need for innovative processes to identify more effective treatment. In line with the repositioning strategy, biological effects of selected benzimidazole carbamates were already investigated in melanoma models. Doudican et al. focused on potential mebendazole activity in chemoresistant M-14 and SK-Mel-19 cell lines. He proved that this compound effectively inhibited the growth and proliferation of malignant melanocytes (IC50 was $0.32 \mu \mathrm{M}$ ) while having a minimal effect on the normal melanocytes. Mebendazole effects were mediated via induced phosphorylation of BCL-2 which contributed to the proapoptotic signaling and resulting cell death. In the light of fact that BCL-2 is widely expressed in human melanoma with a recognized role in melanoma chemoresistance, this discovered mebendazole's activity is of particular interest and deserves further examination (29). Supporting this conclusion is also the fact that mebendazole treatment decreases expression levels of another key antiapoptotic protein $\mathrm{X}$-linked inhibitor of apoptosis (XIAP) both in vitro and in human xenographt melanoma model. This inhibition may occur partially through proteasomal degradation of XIAP and/or via stimulated interaction of SMAC/DIABLO with XIAP (38).

Our of results on antiproliferative activity of flubendazole obtained from model melanoma cell lines Bowes, A-375 and RPMI-7951 generally concur with the findings of Michaelis et al. although our determined IC50 values were generally higher than those reported in their employed melanoma models. We also proved flubendazole-dependent stimulation of mitotic catastrophe with resulting caspase-dependent cell death in all exposed melanoma cells, however, unlike in case of mebendazole with var- ying contribution of individual signaling pathways and negligible involvement of BCL-2 and XIAP proteins. These results clearly suggest that flubendazole may interfere with a variety of signaling pathways and/or targets which among other factors reflects genotypic and phenotypic differences in melanoma cell populations and warrants more detailed further studies.

\section{ANTICANCER ACTIVITY OF BENZIMIDAZOLE CARBAMATES IN CLINICAL STUDIES}

So far, no clinical study on flubendazole effects in human malignancies has been conducted. Still, there exists some clinical information on other members of benzimidazole carbamate family. A pilot study on albendazole in 7 patients with advanced hepatocellular cancer or colorectal cancer with hepatic metastases refractory to other forms of therapy was conducted over 28 days. In patients who received albendazole at $10 \mathrm{mg} / \mathrm{kg} /$ day orally in two divided doses tumor markers (carcinoembryonic antigen or alpha-feto protein) were measured and hematological as well as biochemical parameters were obtained to monitor potential bone marrow, kidney or liver toxicity. Results of this study confirmed a good tolerance of albendazole in all patients with only concern being severe neutropenia in three of them. Importantly, albendazole treatment resulted in decrease of followed tumor markers in two patients while in three others the markers were stabilized, thus demonstrating that albendazole shows antitumor activity in humans (39). In the subsequent dose-finding phase I study of oral albendazole in patients with refractory solid tumors thirty-six patients received doses of $400 \mathrm{mg}$ with dose escalation until 1,200 mg twice a day in a 3 week cycle with serial blood sample collection up to $96 \mathrm{~h}$ and on day 8 of cycles 1 and 4 . The results of this study confirmed that albendazole was well tolerated on the tested treatment schedule, with main dose-limiting toxicity being myelosuppression. Authors recommended the dose of 1,200 mg albendazole twice daily for 14 days in a 21-day cycle for further study (40).

Currently, two clinical trials of mebendazole in brain tumors are carried out. The first one is a phase I open label study in newly diagnosed high-grade glioma patients receiving temozolomide. Patients are treated on a 28 day cycle of $500 \mathrm{mg}$ oral mebendazole three times a day. The primary aim is to determine the maximum tolerated dose of mebendazole with temozolomide and to determine whether this combined regimen can slow tumour progression. The study was completed in September 2016 with no results reported yet. The second clinical trial is phase I and II pilot study of mebendazole in combination with vincristine, carboplatin, and temozolomide. The mebendazole dose is $100 \mathrm{mg}$ twice a day during 70 weeks of treatment. The primary objective of the phase I is verify tolerability of mebendazole dose with the current three-drug regimen. For the phase II aims include time of progression-free status in patients and their overall survival. The study is currently recruiting participants and estimated study completion date is April 2020 (41). 


\section{CONCLUSION}

There is ample evidence that several benzimidazole carbamates and in particular flubendazole and even to a higher degree albendazole and mebendazole show anti-tumor potential in vitro, in vivo, and in silico. Despite the fact that all of them primarily target microtubular system, their concrete mechanisms of action are slightly different. One explanation of this variability might relate to their structural similarity with nucleotides which enables their interaction with a variety of biomolecules. Resulting is the diverse range of mechanisms of action such as reduction of fumarate or glucose uptake in addition to their interaction with cytoskeleton which en face with often chemoresistant and aggressive tumor cells could represent natural advantage. Another strong advantage concerning the potential use of flubendazole as well as other two more intensively studied benzimidazole carbamates is their desmontrated synergism with several clinically approved drugs. To this end published data from clinical trials albeit very limited and preliminary strongly argue in favor of these compounds with well-established pharmacokinetics, excellent toxicity profile and low-cost and warrant their individual further evaluation in oncology.

\section{ACKNOWLEDGEMENTS}

This work was supported by the projects PRVOUK P37/01 and SVV 2016 of Charles University, Faculty of Medicine in Hradec Králové.

\section{REFERENCES}

1. Kato S, Moulder SL, Ueno NT, et al. Challenges and perspective of drug repurposing strategies in early phase clinical trials. Oncoscience 2015 Jun $30 ; 2(6)$ : 576-80.

2. Shim JS, Liu JO. Recent Advances in Drug Repositioning for the Discovery of New Anticancer Drugs. International Journal of Biological Sciences 2014; 10(7): 654-63.

3. Hurle MR, Yang L, Xie Q, Rajpal DK, Sanseau P, Agarwal P. Computational Drug Repositioning: From Data to Therapeutics. Clinical Pharmacology \& Therapeutics 2013 Apr 1; 93(4): 335-41.

4. Mackenzie CD, Geary TG. Flubendazole: a candidate macrofilaricide for lymphatic filariasis and onchocerciasis field programs. Expert Rev Anti Infect Ther 2011 May; 9(5): 497-501.

5. Li YY, Jones SJ. Drug repositioning for personalized medicine. Genome Med. 2012 Mar 30; 4(3): 27.

6. Lacey E. Mode of action of benzimidazoles. Parasitol Today (Regul Ed) $1990 \mathrm{Apr} ; 6(4): 112-5$.

7. Ireland CM, Gull K, Gutteridge WE, Pogson CI. The interaction of benzimidazole carbamates with mammalian microtobule protein. Biochem Pharmacol 1979 Sep 1; 28(17): 2680-2.

8. Cooper GM. Microtubules 2000; Available from: http://www.ncbi. nlm.nih.gov/books/NBK9932/

9. Jordan MA, Wilson L. Microtubules as a target for anticancer drugs. Nat Rev Cancer 2004 Apr; 4(4): 253-65.

10. Jasra N, Sanyal SN, Khera S. Effect of thiabendazole and fenbendazole on glucose uptake and carbohydrate metabolism in Trichuris globulosa. Vet Parasitol 1990 Mar; 35(3): 201-9.

11. Cumino AC, Elissondo MC, Denegri GM. Flubendazole interferes with a wide spectrum of cell homeostatic mechanisms in Echinococcus granulosus protoscoleces. Parasitology International 2009 Sep; $58(3): 270-7$

12. Bossche HV, Thienpont D, Janssens PG. Chemotherapy of Gastrointestinal Helminths. Springer Science \& Business Media 2012. 732 p.

13. Michiels M, Hendriks R, Heykants J, van den Bossche $H$. The pharmacokinetics of mebendazole and flubendazole in animals and man. Arch Int Pharmacodyn Ther 1982 Apr; 256(2): 180-91.

14. European Medicines Agency (online) - Committee for products for veterinary use - flubendazol - Summary report (July 2006). Avail- able at: http://www.ema.europa.eu/docs/en_GB/document_library/ Maximum_Residue_Limits_-_Report/2009/11/WC500014292.pdf. (14 July 2016 , date last accessed).

15. Institute for state control of veterinary biologicals and medicines Summary of Product Characteristics - Flubenol ${ }^{\circledR}$ (online). Available at: http://www.uskvbl.cz. (14 July 2016, date last accessed).

16. McKellar QA, Galbraith EA, Baxter P. Oral absorption and bioavailability of fenbendazole in the dog and the effect of concurrent ingestion of food. J Vet Pharmacol Ther 1993 Jun; 16(2): 189-98.

17. Ceballos L, Elissondo M, Bruni SS, Denegri G, Alvarez L, Lanusse C. Flubendazole in cystic echinococcosis therapy: pharmaco-parasitological evaluation in mice. Parasitol Int 2009 Dec; 58(4): 354-8.

18. Van Leemput L, Heykants J (1991). Flubendazole: concentrations in plasma and residues in edible tissues of pheasants after a 7-day treatment at 60 ppmin the feed. Unpublished report number R 17889/ FK1071. Submitted to FAO by Janssen Pharmaceutica, Beerse, Belgium.

19. Roche G, Canton P, Gérard A, Dureux JB. Treatment of alveolar echinococcosis with flubendazole. Pharmacological study (author's transl.). Pathol Biol 1982 Jun; 30(6): 452-7.

20. Lassègue A, Estavoyer JM, Minazzi H, et al. Treatment of human alveolar echinococcosis with flubendazole. Clinical, morphological and immunological study. Gastroenterol Clin Biol 1984 Apr; 8(4):314-20.

21. Pourgholami MH, Woon L, Almajd R, Akhter J, Bowery P, Morris DL. In vitro and in vivo suppression of growth of hepatocellular carcinoma cells by albendazole. Cancer Letters 2001 Apr 10; 165(1): 43-9.

22. Králová V, Hanušová V, Staňková P, Knoppová K, Čáňová K, Skálová L. Antiproliferative effect of benzimidazole anthelmintics albendazole, ricobendazole, and flubendazole in intestinal cancer cell lines. Anticancer Drugs 2013 Oct; 24(9): 911-9.

23. Pourgholami MH, Akhter J, Wang L, Lu Y, Morris DL. Antitumor activity of albendazole against the human colorectal cancer cell line HT-29: in vitro and in a xenograft model of peritoneal carcinomatosis. Cancer Chemother Pharmacol 2004 Nov 23; 55(5): 425-32.

24. Khalilzadeh A, Wangoo KT, Morris DL, Pourgholami $\mathrm{MH}$. Epothilone-paclitaxel resistant leukemic cells CEM/dEpoB300 are sensitive to albendazole: Involvement of apoptotic pathways. Biochemical Pharmacology 2007 Aug 1; 74(3): 407-14.

25. Chu SWL, Badar S, Morris DL, Pourgholami MH. Potent inhibition of tubulin polymerisation and proliferation of paclitaxel-resistant 1A9PTX22 human ovarian cancer cells by albendazole. Anticancer Res 2009 Oct; 29(10): 3791-6.

26. Martarelli D, Pompei P, Baldi C, Mazzoni G. Mebendazole inhibits growth of human adrenocortical carcinoma cell lines implanted in nude mice. Cancer Chemother Pharmacol 2008 Apr; 61(5): 809-17.

27. Sasaki J, Ramesh R, Chada S, Gomyo Y, Roth JA, Mukhopadhyay T. The anthelmintic drug mebendazole induces mitotic arrest and apoptosis by depolymerizing tubulin in non-small cell lung cancer cells. Mol Cancer Ther 2002 Nov; 1(13): 1201-9.

28. Sawanyawisuth K, Williamson T, Wongkham S, Riggins GJ. Effect of the antiparasitic drug mebendazole on cholangiocarcinoma growth. Southeast Asian J Trop Med Public Health 2014 Nov; 45(6): 1264-70.

29. Doudican N, Rodriguez A, Osman I, Orlow SJ. Mebendazole induces apoptosis via Bcl-2 inactivation in chemoresistant melanoma cells. Mol Cancer Res 2008 Aug; 6(8): 1308-15.

30. Nygren P, Fryknäs M, Agerup B, Larsson R. Repositioning of the anthelmintic drug mebendazole for the treatment for colon cancer. Journal of Cancer Research and Clinical Oncology 2013 Dec; 139(12): 2133-40.

31. Nygren P, Larsson R. Drug repositioning from bench to bedside: tumour remission by the antihelmintic drug mebendazole in refractory metastatic colon cancer. Acta Oncol 2014 Mar; 53(3): 427-8.

32. Pinto LC, Soares BM, Pinheiro J de JV, Riggins GJ, Assumpção PP, Burbano RMR, et al. The anthelmintic drug mebendazole inhibits growth, migration and invasion in gastric cancer cell model. Toxicol In Vitro 2015 Dec; 29(8): 2038-44.

33. Spagnuolo PA, Hu J, Hurren R, et al. The antihelmintic flubendazole inhibits microtubule function through a mechanism distinct from Vinca alkaloids and displays preclinical activity in leukemia and myeloma. Blood 2010 Jun 10; 115(23): 4824-33.

34. Michaelis M, Agha B, Rothweiler F, et al. Identification of flubendazole as potential anti-neuroblastoma compound in a large cell line screen. Sci Rep 2015; 5: 8202.

35. Hou Z-J, Luo X, Zhang W, et al. Flubendazole, FDA-approved anthelmintic, targets breast cancer stem-like cells. Oncotarget $2015 \mathrm{Mar}$ $20 ; 6(8): 6326-40$.

36. Zhang L, Guo M, Li J, et al. Systems biology-based discovery of a potential Atg4B agonist (Flubendazole) that induces autophagy in breast cancer. Mol Biosyst 2015 Nov; 11(11): 2860-6.

37. Králová V, Hanušová V, Rudolf E, Čáňová K, Skálová L. Flubendazole 
induces mitotic catastrophe and senescence in colon cancer cells in vitro. J Pharm Pharmacol 2016 Feb; 68(2): 208-18.

38. Doudican NA, Byron SA, Pollock PM, Orlow SJ. XIAP downregulation accompanies mebendazole growth inhibition in melanoma xenografts. Anticancer Drugs 2013 Feb; 24(2): 181-8.

39. Morris DL, Jourdan JL, Pourgholami MH. Pilot study of albendazole in patients with advanced malignancy. Effect on serum tumor markers/high incidence of neutropenia. Oncology 2001; 61(1): 42-6.
40. Pourgholami MH, Szwajcer M, et al. Phase I clinical trial to determine maximum tolerated dose of oral albendazole in patients with advanced cancer. Cancer Chemother Pharmacol 2010 Feb; 65(3): 597-605.

41. Pantziarka P, Bouche G, Meheus L, Sukhatme V, Sukhatme VP. Repurposing Drugs in Oncology (ReDO) - mebendazole as an anti-cancer agent. Ecancermedicalscience $2014 \mathrm{Jul} 10 ; 8$. 